\title{
Preperitoneal laparoscopic lateral repair in pelvic organ prolapse - a novel approach
}

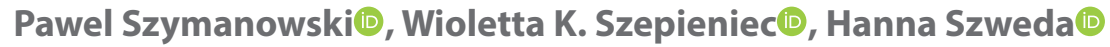 \\ Andrzej Frycz Modrzewski Krakow University, Faculty of Medicine and Health Sciences, Cracow, Poland
}

\begin{abstract}
Objectives: The aim of this study is to present a novel approach for a paravaginal defect treatment. This extraperitoneal approach can be performed in patients with comorbidities and on obese patients. The main advantages are: not requiring the pneumoperitoneum and the Trendelenburg position and the avoidance of peritoneal adhesions.

Material and methods: This study presents the results in 27 patients with cystocele caused by a lateral defect pelvic organ prolapse quantification (POP Q) stage Il or higher. The procedure was performed with a modified Richardson and Burch technique using a preperitoneal approach. Three follow-up examinations were conducted two, six weeks, and six months after the operation. A quality of life assessment was conducted before and after surgery using the short form of the PFIQ-7.

Results: All patients had a POP Q II cystocele and 59\% had concomitant stress urinary incontinence. In all patients cystocele was reduce to asymptomatic POP Q stage I or 0 . Mean operation time was approximately 80 minutes. In six months post-operation follow up, one case of recurrence was noted. The patients' quality of life revealed a statistical improvement from an average of 6.8 points before, to an average of 0.7 points after the operation $(p<0.05)$ in the PFIQ-7.

Conclusions: Preperitoneal laparoscopic lateral repair is a relatively fast procedure and it is also feasible for obese women and for patients with a cardiopulmonary risk. Neither the Trendelenburg position nor the pneumoperitoneum are required. Postoperatively, the patients witnessed a reduction of the cystocele and complaints connected with their previous condition. Key words: lateral defect; Richardson operation; paravaginal repair; Burch operation; preperitoneal approach
\end{abstract}

Ginekologia Polska 2021; 92, 10: 689-694

\section{INTRODUCTION}

Upon vaginal examination pelvic organ prolapse (POP) can be detected in up to $50 \%$ of women and $3-6 \%$ have symptoms related to POP [1]. The primary objective of prolapse surgery is the re-establishment of the pelvic floor anatomy, restoration of the physiological functions of both the bladder and rectum and the recovery of sexual functions. The cause of a paravaginal defect in the anterior compartment, and level II according to de Lancey's classification [2], is the detachment of the vesicovaginal fascia from the arcus tendineus fasciae pelvis. During a gynecological examination using Kristeller speculas, a case of cystocele caused by a lateral defect is seen as a prolapse of the anterior vaginal wall with the preservation of the vaginal rugae. This defect accounts for 60 to $80 \%$ of anterior compartment prolapse [3]. The most typical complaints are discomfort, the sensation of a foreign body and pollakisuria. The patients also frequently suffer from recurrent urinary tract infections connected to urinary retention despite pharmacological treatments, accompanied by urge symptoms.

Richardson et al. [4] first described the anatomy and developed the initial abdominal technique to repair this paravaginal defect. The laparoscopic approaches currently in use have success rates ranging from 60 to $89 \%$ [5]. Until now, the literature has described a transperitoneal laparoscopic approach. Trendelenburg positioning and pneumoperitoneum are required in order to perform such an operation. Consequently this procedure cannot be performed on many patients with cardiopulmonary risk factors.

Richardson [4] also demonstrated the causality of stress urinary incontinence due to a paravaginal defect. Thus, it is common to perform a lateral repair procedure along with the Burch operation. When dealing with a level II defect of pelvic organ prolapse, it should be noted that it commonly occurs along with a level III defect, which causes urinary stress incontinence [6]. However, when there is a level III

\section{Corresponding author:}

Hanna Szweda

Andrzej Frycz Modrzewski Krakow University, Faculty of Medicine and Health Sciences, Cracow, Poland

e-mail:hanna.szweda@gmail.com 
defect along with a level II defect due to urethra kinking it can also mask urinary stress incontinence. It would be revealed, if a Burch operation is not performed simultaneously with the lateral repair procedure.

During the modified Richardson procedure the lateral vaginal wall is lax sutured to the pectineal ligament on the pubic bone which results in the adherence of the vaginal wall to its damaged attachment on the arcus tendineus. The Burch operation is also performed to attach the pubourethral ligaments under the urethra to the pubic bone. The whole procedure is performed without tension. The main process leading to recovery and the re-establishment of the anatomy is the creation of scar tissue between the vaginal wall and arcus tendineus.

Currently, the most common approach for paravaginal repair is the laparoscopic transperitoneal approach. Several papers, however, have described a preperitoneal approach for the Burch procedure $[7,8]$. Taking this into consideration, the authors have developed a novel extraperitoneal technique for paravaginal repair. More accurately, we call it a preperitoneal approach.

When assessing the patient's suitability for this procedure scarring in the abdominal wall is a relative contraindication. The most common procedures which might potentially create difficulties are cesarean sections and an open appendectomy. Scarring after these procedures can complicate the preparation to such a degree that the preperitoneal approach is not feasible and perforation of the peritoneum leads to the transperitoneal approach being used instead.

Presented here is a novel approach to paravaginal repair by using an extraperitoneal (preperitoneal) route. Based on indications and results, this approach might be a viable option for premenopausal women with a cystocele with a lateral defect. It can also be considered for elderly women who have the same defect instead of using anterior mesh implantation. A modified Burch procedure was included as a part of this operation and performed regardless of the complaints of urinary stress incontinence.

\section{MATERIAL AND METHODS}

The study presented here was approved by the local IRB. Patients were recruited from March 2017 to March 2019.

The patients were examined by two surgeons using the Pelvic Organ Prolapse-Quantification (POP-Q) assessment [9]. The inclusion criteria was a symptomatic anterior wall prolapse caused by lateral defect stage POP Q 2 or higher both with and without urethrocele. Exclusion criteria were: a history of previous pelvic surgery or vaginal surgery, genital or abdominal cancer, previous sling operations, patients with concomitant apical defect stage > POP Q I, rectocele or enterocoele, or preoperative post-void residual urine $>50 \mathrm{~mL}$.
A quality of life assessment was conducted before surgery and six weeks after using the short form of the Pelvic Floor Impact Questionnaire (PFIQ-7) [10]. This form evaluates the influence of the disorder on a woman's social life as connected to her urinary tract (UIQ-7), bowel or rectal (CRAIQ-7), and vaginal or pelvic (POPIQ-7) symptoms.

The patients were informed both verbally and in writing of the different procedures including both intra- or extraperitoneal approaches. They were informed that the planned extraperitoneal approach would be substituted for a different operation in the case of perforation of the peritoneum or in the case of straitened preperitoneal preparation.

Before discharge, the postvoid residual (PVR) volume of urine was measured by vaginal ultrasound in patients who reported difficulty during micturition [11].

In cases of post-void residual urine over $80 \mathrm{~mL}$ PVR was checked again after one week. In cases of post-void residual urine over $200 \mathrm{~mL}$ a Foley catheter was inserted for 3 or 4 days. Further management depends on the chronicity of the retention.

Obese women were also included in the present study and as a means of classifying obesity the Body Mass Index (BMI) was used (BMI above 30) [12].

\section{Preoperative considerations}

Before hospital admission every patient was instructed regarding NPO [13].

The patients were allowed to eat up to six hours and drink clear liquids up to two hours before surgery as a means to prevent complications connected with aspiration X.

Compression stockings were worn during the operation.

\section{Operation}

A subumbilical incision is made in the skin. After creating a tent by the operating surgeon and assistant holding the abdominal wall, a trocar is introduced parallel to the skin so that the surgeon can stay in the preperitoneal layer. The preperitoneal space is now filled with $\mathrm{CO} 2$ and a camera is inserted. More blunt preparation is carried out by using the camera and after this preparation three more trocars are introduced. The trocars are placed on both sides of the abdominal wall, two on the left side of the patient $(10 \mathrm{~mm}$ and $5 \mathrm{~mm}$ trocars) for the surgeon, and one on the right side ( $5 \mathrm{~mm}$ trocar) for the assistant. The air pressure is set to $12 \mathrm{~mm} \mathrm{Hg}$ with an air flow rate of $6 \mathrm{~mL} / \mathrm{sec}$. The patient position stays horizontal without Trendelenburg positioning.

It should be noted here that the preparation is quite simple even in obese women because the distribution of fat is small in the preperitoneal space, thus making it feasible even for overweight women. The retropubic space is now enlarged and the bladder moved medially to reach the vaginal wall. When the vaginal wall is reached, a non-resorbable 
braided 0 suture, $15 \mathrm{~cm}$ long is introduced into the retropubic cavity. The surgeon inserts the left forefinger into the vagina and with this finger moves the vaginal wall closer to the pubic bone and with the right hand places the suture in such a way as to have control of the vaginal wall while ensuring not to perforate it. After the placement of this suture the surgeon changes gloves and an assistant holds the vaginal wall close to the pubic bone so that the suture can be tied without tension (Fig. 1). The vaginal wall is sutured to the medial part of Cooper's ligament with two non-resorbable, braided 0 sutures. This type of suture results in the vaginal wall contacting the arcus tendineus (Fig. 2). The next suture is placed paraurethral under the middle aspect of the urethra. This is a modification of the Burch operation in which two sutures are used. The Burch suture is placed under low to medium tension so that there is no hypercorrection. The appearance of the sutures after the operation resembles the lines of a hammock. The procedure is now repeated on the other side (Fig. 3). After hemostasis is achieved Redon drain is placed in the preperitoneal space and all of the instruments and trocars are removed. The incisions are closed with resorbable, monofilament 4-0 sutures.

\section{Postoperative considerations}

The patient can drink fluids immediately after the operation and eat after approximately 4-6 hours. Thromboprophylaxis is achieved with low-weight heparin and compression stockings. Patient mobilization can take place in the evening of the day of the operation while the bladder catheter is usually kept in place until the next morning. The patient can be discharged the following day. Three

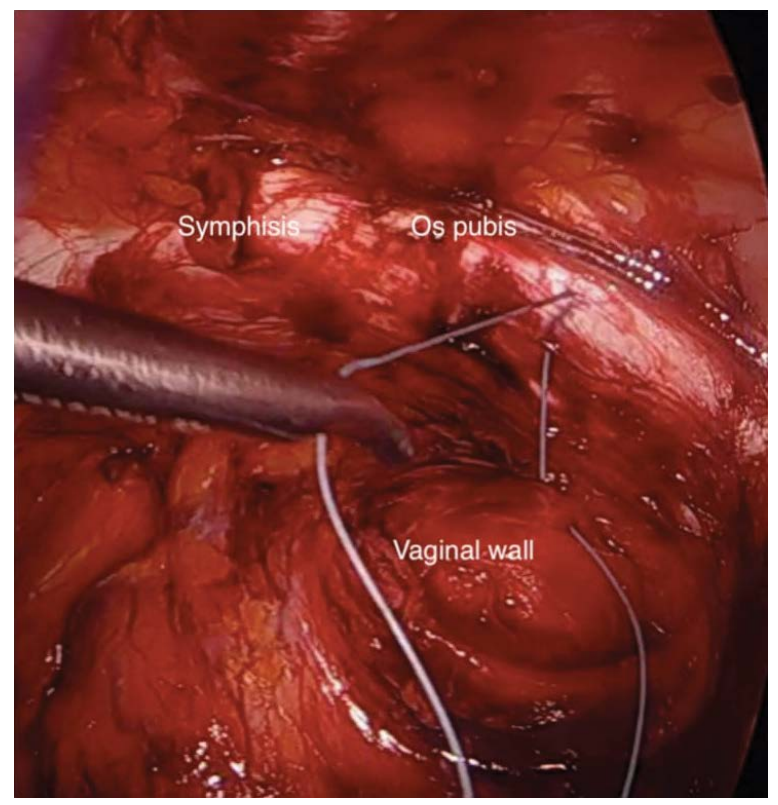

Figure 1. Fixation of the vaginal wall to the arcus tendineus fasciae pelvis follow-up examinations measuring urine retention are usually conducted two weeks, six weeks and six month after the operation.

The patient is asked not to lift or carry heavy objects and should also refrain from doing any hard work for approximately 6 weeks after the operation. The patient is also shown the correct position to sneeze or cough with the head turned as far as possible to the left or right side of the body for a better distribution of pressure to protect the reconstructed pelvic floor [14].

\section{RESULTS}

All of the 27 patients had a mean age of 43 (31-57), 89\% were premenopausal, with a level II lateral defect in anterior compartment (cystocele). Three obese women (BMI over 30) were included in this study (Tab. 1). Fifty-nine percent complained of stress urinary incontinence. This complaint

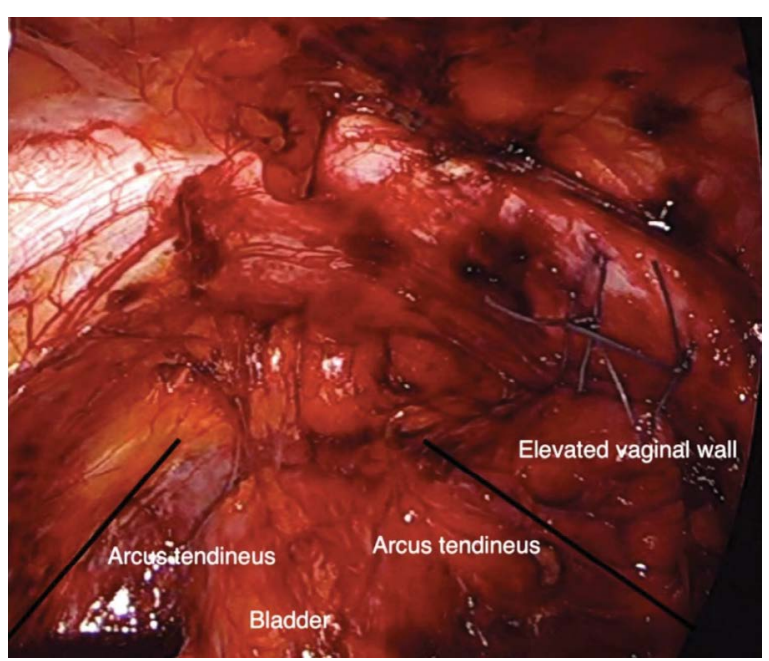

Figure 2. Vaginal wall fixed to the arcus tendineus fasciae pelvis

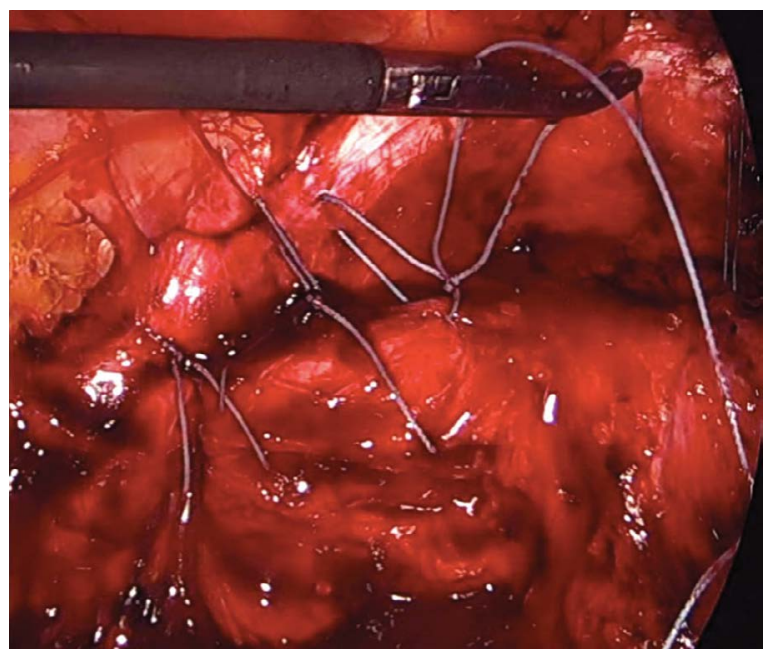

Figure 3. Lax fixation of the paraurethral area - modified Burch suture 
Table 1. General characterisctics of the study group / Demographic and medical description of the study population

\begin{tabular}{|l|l|}
\hline Number of patients & $\mathbf{n}=\mathbf{2 7}$ \\
\hline Age/Age range & Mean 43 years/31-57 years \\
\hline BMI/BMI range value & Mean 25/17-34 \\
\hline $\mathrm{BMI}>30$ & $3(11.1 \%)$ \\
\hline Smoking & $6(22 \%)$ \\
\hline Premenopausal & $24(89 \%)$ \\
\hline Parity/Parity range & Mean $2 / 1-3$ \\
\hline Weight of biggest child/range & Mean 3680 grams/2700-4700 grams \\
\hline Family POP history & $11(40.7 \%)$ \\
\hline
\end{tabular}

$\mathrm{BMI}$ - body mass index

\begin{tabular}{|l|l|}
\hline Table 2. Post Operative Complications \\
\hline Number of patients & $\mathbf{n = 2 7}$ \\
\hline Operating time/range & $\begin{array}{l}\text { Mean } 80 \text { minutes/ } \\
/ 55-130 \text { minutes }\end{array}$ \\
\hline postoperative pain till 14 days & $1(3.7 \%)$ \\
\hline Urine retention ( $>100 \mathrm{~mL}$ after voiding) & $2(7 \%)$ \\
\hline POP recurrence (6 month observation time) & $1(3.7 \%)$
\end{tabular}

was confirmed in a cough test. After the operation the lateral defect was reduced to POP Q 0 or I in all patients. In this type of operation is important to forestall hypercorrection which can lead to urine retention. Using the presented procedure, reducing the cystocele to at least POP Q I seems to be sufficient to eliminate the ailments. In patients with POP Q I cystocele no urge symptoms, urinary incontinence or other complaints were observed in the study group. Overall, the operation was very well-tolerated.

The most common postoperative complication was urine retention (more than $100 \mathrm{~mL}$ after voiding). This complication occurred in two patients (7\%) and was only a temporary problem which resolved itself completely after a maximum time of four weeks. Only one patient complained of postoperative pain (3.7\%) and the objective pain severity was six in the VAS-Scale (VAS, visual analogue scale). One patient (3.7\%) experienced a recurrence of the symptoms with a lateral cystocele six months after the operation. As a postmenopausal woman, this patient was scheduled to anterior colporrhaphy using alloplastic material (Tab. 2).

The mean operation time was 80 minutes and the patients were discharged from the hospital the day after the operation. An ultrasound was performed only when the patient complained of a postoperative problem with micturition.

Quality of life was assessed using the PFIQ-7 (PFIQ, pelvic floor impact questionnaire). Overall it revealed an average postoperative improvement from 6.8 to $0.7(p<0.05)$. For the anterior compartment there was an improvement on average from 11.7 to $1.2(p<0.05)$.

\section{DISCUSSION}

Repairing a lateral defect using this preperitoneal technique makes it feasible for almost all women, regardless contraindications for classic laparoscopy. In this study three of the patients were obese. It was observed that the distribution of fat in the preperitoneal space is relatively slight, making the preparation simple and fast in almost all body types.

By eliminating the Trendelenburg position this approach is also feasible for women with cardiopulmonary, ophthalmological and neurological problems. It has been shown in the literature that cardiac output is not influenced to a great degree by this position, but that intraocular pressure is significantly raised [15]. Because of potentially life-threatening complications due to the Trendelenburg positioning, especially in cases of a long operating time, the preperitoneal approach can be considered with elderly and also multimorbid patients [16].

Observation in our department shows that most patients after two hours in a head-down position often need a quick re-alignment of their body because of pulmonary and cardiac instability. By performing a preperitoneal operation the body of the patient is kept in a horizontal position and subsequent instability of the patient was not observed in the present study. However, the population of the current study had no cardiological risks so there is no evidence that this approach has any influence on such risks.

Operations with pneumoperitoneum cause an increase in the mean arterial pressure of approximately $30 \%$ and a decrease in venous pressure of about $25 \%$. Because of the compression of the vena cava venous reverse flow is decreased. Other observations included an increase in the level of adrenaline to approximately three times its normal level, cortisol four times, and vasopressin about 40 times, leading to an increase in peripheral pressure [17]. This research was studied on animal models, but it can be presumed to be applicable in a human patient. Some of these changes are caused by carbon dioxide while others are caused by the pressure in the abdominal cavity. The preperitoneal approach prevents pressure changes and complications can be avoided. It also prevents peritoneal adhesions, which can result in postoperative pain or difficulties in future operations. Further studies, however, are needed to evaluate these issues.

The next important advantage of this technique is that no mesh implants are utilized. This is seen as a positive aspect in context of the FDA warning about MESH from 2011 , indicating the seriousness of the problem with alloplastic materials [18]. Alloplastic material in the human body can lead to pain, discomfort, or erosion of the vaginal wall, 
vaginal bleeding and dyspareunia. The use of synthetic implants is increasingly restricted, among others, by FDA [19].

As a reaction for this restrictions, different surgical techniques enabling to minimize or eliminate meshes use in prolapse surgery. A good example is an apical defect reconstruction described by Śliwa et al. [20] in which a minimal portion of alloplastic mesh is used, and in addition is placed extraperitoneally. One of the common indications for the anterior mesh implantation is a cystocele with a lateral defect, especially in elderly women. In this population, a transperitoneal laparoscopic approach is often contraindicated. Although laparoscopic surgery is minimally invasive, it is often a weight bearing procedure for this group of patients which may result in cardiopulmonary problems.

During the preperitoneal lateral repair only non-resorbable sutures and no meshes were used. The cause of a lateral defect is the abruption of the vesico-vaginal fascia from its attachment. The suturing and re-establishment of this attachment is a sufficient therapy for a cystocele with lateral defect. While sutures in and of themselves do not exactly solve this problem it is rather the creation of a scar by the wound healing between the vaginal wall and arcus tendineus which sufficiently reduces this defect. Because of this the use of resorbable sutures will likely lead to the same outcome and should be considered in the future.

The fixation of prolapsed tissues is made as in the originally developed and method [21] and its intraperitoneal laparoscopic modification [22]. Satisfactory long term results of this, anteriorly directed fixation can be extrapolated to our preperitoneal modification of the procedure.

The group of patients in this study consisted of young premenopausal women. Considering the reduction of risk by eliminating the Trendelenburg position this approach can presumably be used likewise in elderly patients. This thesis, however, needs to be proven in future studies.

Chinthakanan et al. [5] describes high success rates in the range of $60 \%$ to $89 \%$ after performing laparoscopic paravaginal defect repair. According to him, laparoscopic paravaginal repair approaches should be performed as a first-line therapy, especially in the age of laparoscopic and robotic surgery. He also points out that anterior colporrhaphy is not an anatomic repair for paravaginal defects.

Rudnicki at al. [23] compared 68 patients after anterior colporrhaphy and 70 patients with collagen-coated transvaginal mesh for anterior vaginal wall prolapse. After a three-year follow-up the anatomical cure rate was $91.4 \%$ in the mesh group and $41.2 \%$ in the colporrhaphy group. Although there was no impact on the subjective outcomes.

This low anatomical rate in the anterior colporrhaphy group can be explained by the fact that anterior colporrhaphy is not a precise treatment for a paravaginal lateral defect [24, 25]. It should be even considered as contraindicated in this clinical situation.

The recurrence rate after laparoscopic preperitoneal lateral repair needs to be proven in long term prospective studies on a major group of patients. If the rate is comparable to anterior mesh implantation and the stress is bearable for elderly patients, this procedure can potentially be an alternative for mesh implantation in treatment of a cystocele caused by lateral defect. In this way the use of mesh may be reduced the cases of recurrence.

The next advantage of this procedure is that the laparoscopic approach does not cause a narrowing of the vagina which is also an important factor for young women preventing dyspareunia which can occur after vaginal operations, especially after mesh implantation [23].

Limitations of this study are a short 6-month observation time and a small patient group. A larger study group and longer observation time are needed for more conclusions.

Limitations of this procedure are any anatomical changes, especially scar tissue after previous operations, in the preperitoneal space.

\section{CONCLUSIONS}

Preperitoneal laparoscopic lateral defect repair can be done in a relatively short time. It is also feasible for obese women and for patients with cardiopulmonary risks, because the Trendelenburg position and pneumoperitoneum are both unnecessary. Any synthetic meshes are not administered. A very satisfactory effect can be achieved with a lessening of patient's complaints and reduction of the cystocele.

The first short-term results of this preperitoneal procedure show that this procedure is fast, simple, and the correction of the defect is satisfactory for the patients. Further studies are needed for a better assessment of the results and the risk of recurrence.

\section{Conflict of interest}

The authors state that there are no conflicts of interest to disclose.

\section{REFERENCES}

1. Barber $M$, Maher C. Epidemiology and outcome assessment of pelvic organ prolapse. International Urogynecology Journal. 2013; 24(11): 1783-1790, doi: 10.1007/s00192-013-2169-9.

2. Perucchini $D$, De Lancey JO. Functional anatomy of the pelvic floor and lower urinary tract. In: Baessler K, Schüssler B, Burgio KL, Moore $\mathrm{K}$, Stanton SL. ed. Pelvic floor re-education. Principles and practice. Springer 2008: 3-21.

3. Hosni MM, El-Feky AEH, Agur WI, et al. Evaluation of three different surgical approaches in repairing paravaginal support defects: a comparative trial. Arch Gynecol Obstet. 2013; 288(6): 1341-1348, doi: 10.1007/s00404013-2927-4, indexed in Pubmed: 23797932.

4. Richardson AC, Edmonds PB, Williams NL. Treatment of stress urinary incontinence due to paravaginal fascial defect. Obstet Gynecol. 1981; 57(3): 357-362, indexed in Pubmed: 7465150. 
5. Chinthakanan O, Miklos JR, Moore RD. Laparoscopic paravaginal defect repair: surgical technique and a literature review. Surg Technol Int. 2015; 27: 173-183, indexed in Pubmed: 26680393.

6. Petros PE, Ulmsten UI. An integral theory of female urinary incontinence. Experimental and clinical considerations. Acta Obstet Gynecol Scand Suppl. 1990; 153: 7-31, doi: 10.1111/j.1600-0412.1990.tb08027.x, indexed in Pubmed: 2093278.

7. Tiras MB, Sendag F, Dilek U, et al. Laparoscopic burch colposuspension: comparison of effectiveness of extraperitoneal and transperitoneal techniques. Eur J Obstet Gynecol Reprod Biol. 2004; 116(1): 79-84, doi: 10.1016/j.ejogrb.2004.02.003, indexed in Pubmed: 15294373.

8. von Theobald P, Guillaumin D, Lévy G. Laparoscopic preperitoneal colposuspension for stress incontinence in women. Technique and results of 37 procedures. Surg Endosc. 1995; 9(11): 1189-1192, doi: 10.1007/BF00210925, indexed in Pubmed: 8553231.

9. Barber MD, Lambers A, Visco AG, et al. Effect of patient position on clinical evaluation of pelvic organ prolapse. Obstet Gynecol. 2000; 96(1): 18-22, doi: 10.1016/s0029-7844(00)00859-0, indexed in Pubmed: 10862835.

10. Barber MD, Walters MD, Bump RC. Short forms of two condition-specific quality-of-life questionnaires for women with pelvic floor disorders (PFDI-20 and PFIQ-7). Am J Obstet Gynecol. 2005; 193(1): 103-113, doi: 10.1016/j.ajog.2004.12.025, indexed in Pubmed: 16021067.

11. Serlin DC, Heidelbaugh JJ, Stoffel JT. Urinary retention in adults: evaluation and initial management. Am Fam Physician. 2018; 98(8): 496-503, indexed in Pubmed: 30277739.

12. World Health Organization. Obesity: preventing and managing the global epidemic. Report of a WHO consultation. WHO technical report series 894. , Geneva 2000.

13. Pham $\mathrm{CH}, \mathrm{Collier} \mathrm{ZJ}, \mathrm{Webb} A B$, et al. How long are burn patients really NPO in the perioperative period and can we effectively correct the caloric deficit using an enteral feeding „Catch-up” protocol? Burns. 2018; 44(8): 20062010, doi: 10.1016/j.burns.2018.07.005, indexed in Pubmed: 30115532.

14. Bo K, Berghmans B, Morkved S, van Ka. Evidence-based physical therapy for the pelvic floor 2 nd edition. Bridging science and clinical practice. Churchill Livingstone, London 2014.

15. JoYYi, Kim JiY, Chang YJ, et al. The effect of equal ratio ventilation on oxygenation, respiratory mechanics, and cerebral perfusion pressure during laparoscopy in the trendelenburg position. Surg Laparosc Endosc Percutan Tech. 2016; 26(3): 221-225, doi: 10.1097/SLE.0000000000000276, indexed in Pubmed: 27258912.
16. Assad OM, El Sayed AA, Khalil MA. Comparison of volume-controlled ventilation and pressure-controlled ventilation volume guaranteed during laparoscopic surgery in Trendelenburg position. J Clin Anesth. 2016; 34 55-61, doi: 10.1016/j.jclinane.2016.03.053, indexed in Pubmed: 27687346.

17. Mann C, Boccara G, Pouzeratte $Y$, et al. The relationship among carbon dioxide pneumoperitoneum, vasopressin release, and hemodynamic changes. Anesth Analg. 1999; 89(2): 278-283, doi: 10.1097/00000539199908000-00003, indexed in Pubmed: 10439730.

18. Urogynecologic surgical mesh: update on the safety and effectiveness of transvaginal placement for pelvic organ prolapse. https://www.fda. gov/media/81123/download (2021-02-24).

19. FDA's activities: urogynecologic surgical mesh. https://www.fda. gov/medical-devices/urogynecologic-surgical-mesh-implants/fdas-activities-urogynecologic-surgical-mesh (2021-04-17).

20. Śliwa J, Kryza-Ottou A, Zimmer-Stelmach A, et al. A new technique of laparoscopic fixation of the uterus to the anterior abdominal wall with the use of overfascial mesh in the treatment of pelvic organ prolapse. Int Urogynecol J. 2020; 31(10): 2165-2167, doi: 10.1007/s00192-02004287-4, indexed in Pubmed: 32303776.

21. Heidenreich W. Williams-Richardson vaginopexy. An abdominal suspension operation in prolapse and extensive vaginal descent [article in German]. Zentralbl Gynakol. 1997; 119(8): 378-382, indexed in Pubmed: 9340978.

22. Miklos JR, Kohli N. Laparoscopic paravaginal repair plus burch colposuspension: review and descriptive technique. Urology. 2000; 56(6 Suppl 1): 64-69, doi: 10.1016/s0090-4295(00)00510-0, indexed in Pubmed: 11114565.

23. Rudnicki $M$, Laurikainen $E$, Pogosean $R$, et al. A 3-year follow-up after anterior colporrhaphy compared with collagen-coated transvaginal mesh for anterior vaginal wall prolapse: a randomised controlled trial. BJOG. 2016; 123(1): 136-142, doi: 10.1111/1471-0528.13628, indexed in Pubmed: 26420345

24. Dietz HP, Pang S, Korda A, et al. Paravaginal defects: a comparison of clinical examination and 2D/3D ultrasound imaging. Aust N Z J Obstet Gynaecol. 2005; 45(3): 187-190, doi: 10.1111/j.1479-828X.2005.00377.x, indexed in Pubmed: 15904441.

25. Ubertazzi EP, Soderini HFE, Saavedra Sanchez AJM, et al. Long-term outcomes of transvaginal mesh (TVM) In patients with pelvic organ prolapse: A 5-year follow-up. Eur J Obstet Gynecol Reprod Biol. 2018; 225: 90-94, doi: 10.1016/j.ejogrb.2018.03.060, indexed in Pubmed: 29680466. 